\title{
Single-cell analysis identifies key pathways and prognostic markers in lupus nephritis
}

these findings
might provide
an opportunity
for further
personalization
of therapy

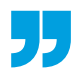

Clinical and histopathological findings in lupus nephritis (LN) are heterogeneous and insufficient for accurate prognosis. Now, Jill Buyon, Thomas Tuschl, Chaim Putterman and colleagues have used single-cell RNA sequencing (scRNA-seq) of kidney and skin biopsy samples to identify molecular signatures that could potentially be used to predict treatment responses and enable personalized therapy in $\mathrm{LN}$.

"ScRNA-seq provides a very detailed view of gene expression at high resolution, enabling exploration of the heterogeneity of cell types and states," explains Evan Der, first author of the study. "A major advantage of this approach was that we could generate data from very small pieces of kidney tissue that were obtained during clinically indicated biopsies in patients with suspected LN."

The researchers analysed kidney biopsy samples from 21 patients with LN and skin biopsy samples from 17 of these patients. They identified upregulation of type I interferon (IFN) response pathway genes in renal tubular cells and skin keratinocytes from the patients compared to those from three healthy individuals. The IFNresponse signature in the tubular

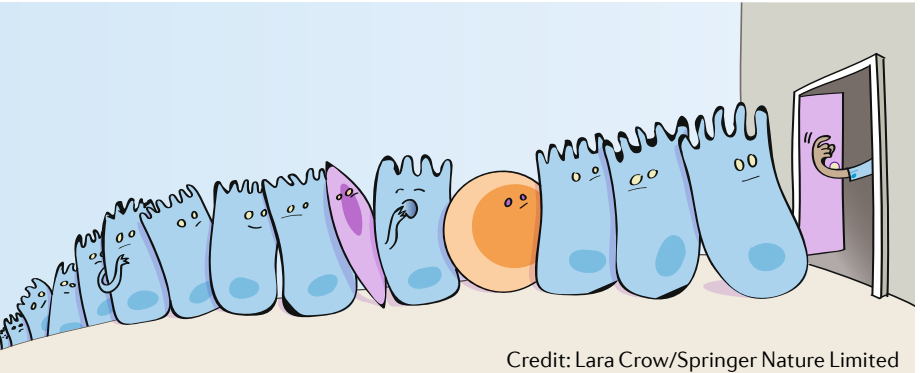

cells was also significantly higher in patients who did not respond to therapy than in those who were partial or complete responders at 6 months after the kidney biopsy. "These data support continuing investigation into how the IFN pathway could lead to scarring in the kidney, which is what we most want to avoid in LN," says Buyon.

Tubular cells from patients who did not respond to therapy also showed significantly higher expression of genes that encode extracellular matrix (ECM) and ECM-interaction proteins reflecting an active fibrotic pathway - than did those from treatment-responsive patients. As this gene expression signature was present in the tubular cells of some patients whose kidney biopsy samples did not show histological evidence of fibrosis, the researchers suggest that it might predict a fibrotic response. They also identified a tubular cell signature of fibrosis comprising four collagen genes that could predict the response to treatment of patients with $\mathrm{LN}$ at 6-months post-biopsy with $92 \%$ accuracy. The keratinocytes of patients who did not respond to therapy also showed upregulation of ECM genes, and the fibrotic gene signature of these cells correlated with that of tubular cells in individual patients.

"Identification of gene expression signatures in the fibrosis and IFN pathways with prognostic significance at the time of kidney biopsy is highly significant because if validated, these signatures could be quickly adapted for patient stratification and personalization of treatment and follow-up," comments
Putterman. "The identification of similar signatures in the skin raises the possibility that this much more accessible organ could provide a window on pathogenic processes happening in the kidney. Using skin as a surrogate source of biomarkers for renal disease could potentially enable more frequent monitoring without the need for repeat renal biopsies."

The researchers also identified several pathways that were differentially expressed in tubular cells and keratinocytes from patients with different histological classes of LN. These included TNF, IL-1 and IFN signalling pathways. They suggest that these findings might provide an opportunity for further personalization of therapy.

"Ultimately, our research could lead to improved prediction of responses to conventional therapies and enable earlier consideration of more aggressive or alternative treatment approaches to improve patient outcomes," concludes Putterman. "Although we know that scarring is important, current therapies for $\mathrm{LN}$ are directed against the inflammatory response," adds Buyon. "I think our findings call into the armamentarium drugs that will stop the scarring process. Dual therapies that target inflammation and fibrosis might be very interesting approaches for the treatment of LN."

Ellen F. Carney

This article is modified from the original in Nat. Rev. Nephrol. (https://doi.org/10.1038/s41581-019-0164-1)

ORIGINAL ARTICLE Der, E. et al. Tubular cell and keratinocyte single-cell transcriptomics applied to lupus nephritis reveal type IIFN and fibrosis relevant pathways. Nat. Immunol. https://doi.org/10.1038/ 541590-019-0386-1 (2019) 